\title{
On the Yellow Product and Browning of the Reaction of Dehydroascorbic Acid with Amino Acids
}

\author{
Tateki HaYASHI, Yasunari HoshII and Mitsuo NAMIKI \\ Department of Food Science and Technology, Faculty of Agriculture, \\ Nagoya University, Chikusa-ku, Nagoya 464, Japan
}

Received September 29, 1982

\begin{abstract}
The ethanolic reaction mixture of dehydroascorbic acid (DHA) and an amino acid contained a significant quantity of a yellow product. During the reaction, this yellow product was found to appear only after the formation of scorbamic acid (SCA) and the red pigment. The reaction of SCA with DHA or with the red pigment (the oxidized form of bis(2-deoxy-2-L-ascorbyl)amine) also produced the same yellow product. Its formation by the DHA-amino acid reaction was supposed to be the direct result of the above SCA-red pigment reaction. The isolated yellow product was examined mainly by spectroscopy, and a possible structure (1) containing one molecular portion each of hydrated DHA, cyclic DHA and AsA residues, and two nitrogen atoms was proposed. The yellow product is probably a condensation and oxidation product of the reaction between SCA and the red pigment. The formation of the yellow product is probably a key intermediate in this kind of browning reaction. This supposition was as also supported by the highest degree of browning shown by the yellow product among other intermediates.
\end{abstract}

The reaction of dehydroascorbic acid (DHA), oxidized ascorbic acid, with various amino acids has been known to cause browning of the mixture, which reaction is said to be related to the process of deterioration of certain kinds of foodstuffs. From such a reaction mixture a red pigment has been isolated and identified to be the oxidized form of bis(2deoxy-2-L-ascorbyl)amine as an intermediate of this reaction. ${ }^{1)}$ In the series of work concerning the amino-carbonyl reactions, we have shown the formation of three different free radical species $\mathrm{R}-\mathrm{A}, \mathrm{R}-\mathrm{B}$ and $\mathrm{R}-\mathrm{C}$ in the reacting mixture. ${ }^{2}$ One of these, $\mathrm{R}-\mathrm{A}$, has been isolated in pure form on a chromatographic plate and proved to be a fairly stable free radical. Its parent compound, tris(2-deoxy-2L-ascorbyl)amine, has been also isolated, and the course of formation of the above free radical from this product by one-electron oxidation has been elucidated. ${ }^{3 \sim 5)}$ During these studies we have been aware that a certain yellow substance is also produced in considerable quantity. The present work is concerned with its isolation and structural study, the path of its formation, and its significance as a key intermadiate of the browning reaction.

\section{RESULTS AND DISCUSSION}

\section{Presence of yellow product in the reaction} mixture of DHA-amino acid

When the mixture of DHA $(0.4 \mathrm{mmol})$ and phenylalanine (Phe $0.2 \mathrm{mmol}$ ) suspended in $2 \mathrm{ml}$ of $95 \%$ ethanol was refluxed, the mixture turned immediately red, which deepened for about $3 \mathrm{~min}$ and then turned brown. Separation of the products in the mixture, which was refluxed for 8 or $25 \mathrm{~min}$, on a cellulose thin-layer plate, showed many spots corresponding to the products (Fig. 1). The red spot at $R f 0.36$ can be identified with the red pigment, which is the oxidized form of bis(2deoxy-2-L-ascorbyl)amine, by comparison with a standard specimen.

The yellow spot found closely above this red spot at $R f 0.40$ has not been examined before, even though it has been observed generally in comparable or larger quantities than that of the red pigment. This spot appeared invariable 
when Ala or Met was used as the starting amino acid as shown in Fig. 1. This suggested that the reaction which produces the yellow product mainly proceeds through the reductive

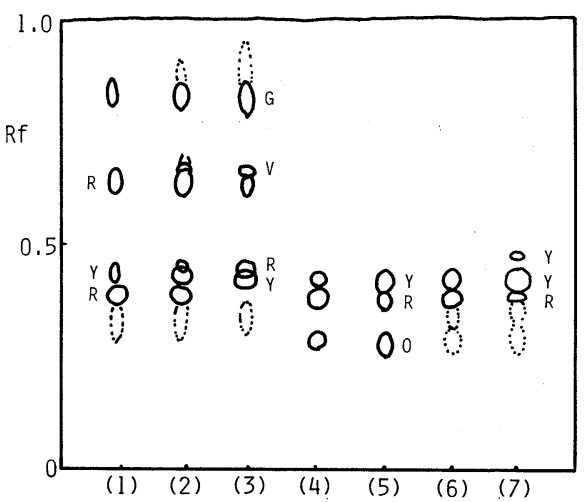

FIG. 1. TLC of the Reaction Mixtures of DHA with Amino Acids in Ethanol.

Mixtures of DHA ( $0.2 \mathrm{M})$ and amino acids ( $0.1 \mathrm{M})$ in $95 \%$ ethanol were refluxed in a boiling water bath. Reaction system: (1) (3), DHA + Phe; (4), (5), DHA + Ala; (6), (7), DHA + Met. Heating time: (1) $3 \mathrm{~min}$; (2), (4), (6) $8 \mathrm{~min}$; (3), (5), (7) $25 \mathrm{~min}$. R, red; Y, yellow; V, violet; G, green. amination of DHA by a Strecker degradation, without participation of the alkyl residue of the amino acid.

The colored spots above $R f 0.5$ appeared only when Phe was employed as the amino acid, and were considered of less importance in the main browning reaction, and hence were not studied in the present work.

\section{Dependence of the formation of the yellow} product on the amino acid species

Table I shows the relative yield of the yellow product by the reaction of DHA with a variety of amino acids. The reaction with Met, Phe or Ala gave a higher yield of this substance than that with Tyr, Asp or His. The absence of the yellow product in the reaction mixture of DHA with Trp was attributed to the much higher rate of the browning reaction with Trp than with other amino acids (see browning in the same table), which probably caused the yellow product to degrade or polymerize at an early stage of the reaction. Amino acids with

Table I. Amount of Products in the Reaction Mixtures of DHA AND Amino AcIDs (0.2 and $0.1 \mathrm{M}$, each)

\begin{tabular}{ccccc}
\hline $\begin{array}{c}\text { Amino } \\
\text { acid }\end{array}$ & $\begin{array}{c}\text { Heating } \\
\text { time } \\
(\mathrm{min})\end{array}$ & Red pigment & Yellow product & $\begin{array}{c}\text { Rrowning } \\
\text { (O.D. at 370 nm) }\end{array}$ \\
\cline { 3 - 4 } Phe & 8 & 1.2 & 2.9 & 0.64 \\
& 25 & 0.0 & 5.8 & 0.74 \\
Ala & 8 & 2.3 & 1.0 & 0.21 \\
& 25 & 0.0 & 4.5 & 0.35 \\
Pro & 8 & 0.0 & 0.0 & 0.99 \\
& 25 & 0.0 & 0.0 & 0.92 \\
Cys & 8 & 0.0 & 0.0 & 0.00 \\
& 25 & 0.0 & 0.0 & 0.06 \\
Met & 8 & 3.5 & 5.0 & 0.51 \\
& 25 & 1.2 & 8.0 & 0.61 \\
Trp & 8 & 1.2 & 0.0 & 0.91 \\
& 25 & 0.0 & 0.0 & 1.40 \\
Tyr & 8 & 0.1 & 0.0 & 0.01 \\
& 25 & 0.3 & 0.9 & 0.16 \\
Asp & 8 & 0.3 & 0.0 & 0.01 \\
& 25 & 0.2 & 0.3 & 0.04 \\
Arg & 8 & 2.6 & 0.0 & 0.15 \\
& 25 & 2.0 & 0.0 & 0.24 \\
His & 8 & 2.6 & 0.2 & 0.19 \\
& 25 & 4.3 & 1.2 & 0.41 \\
Lys & 8 & 0.0 & 0.0 & 0.41 \\
& 25 & 0.0 & 0.0 & 0.93 \\
\hline
\end{tabular}


amino groups other than the $\alpha$-amino groups, Pro, Arg and Lys, did not give the yellow product under the conditions employed. Cys seemed to react hardly at all with DHA, perhaps because of its reducing property.

The amounts of the red pigment and the browning produced are also recorded in the same table. As these values were parallel with those of the yellow product, except for the browning which gave no yellow product, the formation of these two products as well as the browning were considered to be closely related to each other. These facts suggest that the yellow product is one of the key intermadiates of the browning reaction involving DHA.

\section{Formation of the yellow product in the} course of the reaction of DHA with Phe

The variation of the amounts of the yellow product, the red pigment and SCA during the reaction of DHA with Phe was followed by the analytical methods described in the experimental section. Examination of Fig. 2 shows that the yellow product appeared only after the initial increase and subsequent decrease of the

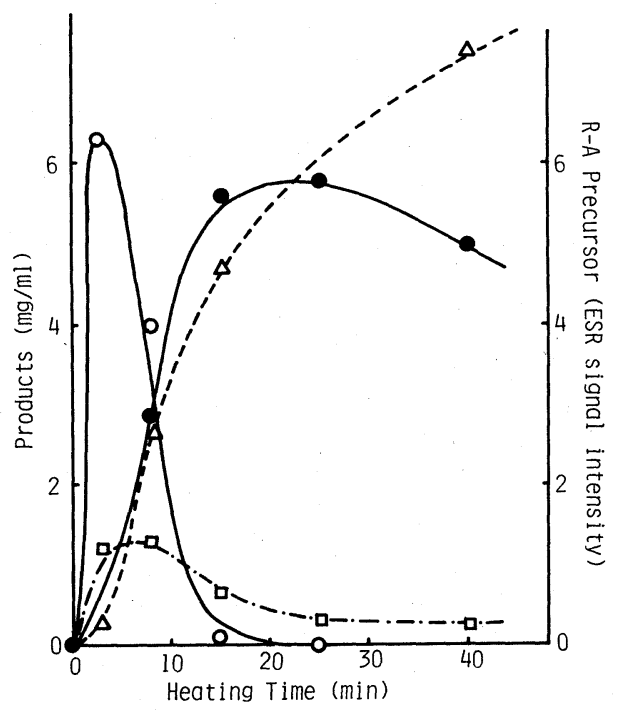

FIG. 2. Formation of Yellow and Other Products during the Reaction of DHA with Phe.

The mixture of DHA $(0.2 \mathrm{M})$ and Phe $(0.1 \mathrm{M})$ in ethanol was refluxed in a boiling water bath. - $\mathrm{O}-$, red pigment; $---\square--$, SCA; - - , yellow product; --- $\triangle---$, R-A precursor. other two products.

Substitution of the $95 \%$ ethanol solvent by methyl cellosolve or dimethyl sulfoxide decreased the yield of the yellow product. By this reaction in water, the yellow product was not detected even though the red pigment was abundant. As will be shown later, this is considered to be due to the readiness of the yellow product to undergo the browning reaction, especially when the solvent is water.

As has already been reported, ${ }^{6}$ the addition of AsA to the DHA-amino acid reaction system has the effect, amongst others, of increasing the yield of the precursor to the free radical R-A, namely tris(2-deoxy-2-L-ascorbyl)amine. Figure 3 shows that AsA also has the effect of significantly decreasing the yield of the yellow product. This may perhaps mean that an oxidative environment is required for the formation of the yellow product.

\section{The formation of the yellow product from other intermediates}

Scorbamic acid (SCA) is known to be one of the initial products of the reaction of DHA with amino acids by condensation followed by Strecker degradation. ${ }^{1)}$ Heating the SCA alone or with AsA did not produce the yellow product, but that with DHA producted the red pigment and then the yellow product. As is seen in Fig. 4 the amount of the yellow product

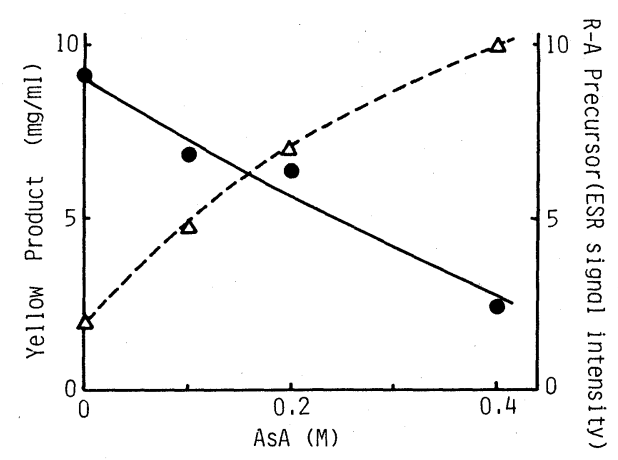

FIG. 3. Effect of AsA Concentration on the Formation of Yellow Product and Its R-A Precursor.

The mixture of DHA $(0.4 \mathrm{M})$, Phe $(0.2 \mathrm{M})$ and each concentration of AsA in ethanol was refluxed for $25 \mathrm{~min}$ in a boiling water bath. - - - yellow product; --- $\triangle---$, R-A precursor. 


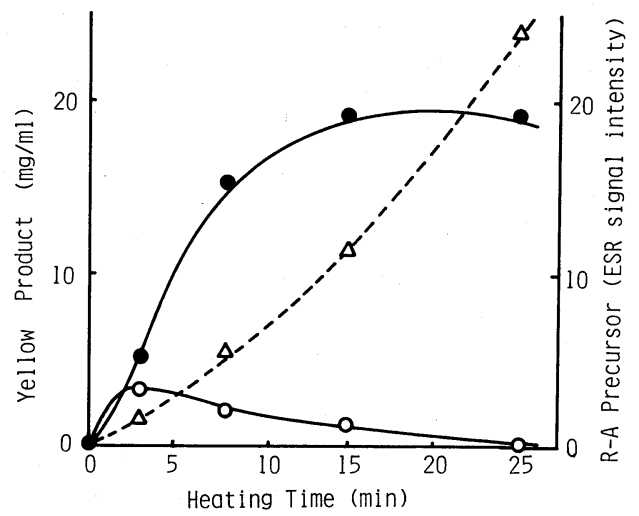

Fig. 4. Formation of Yellow and Other Products by the Reaction of DHA with SCA.

The mixture of DHA and SCA ( $0.2 \mathrm{M}$ each) was refluxed in a boiling water bath. - - - yellow product; $-\mathrm{O}-$, red pigment; --- $\triangle---$, R-A precursor.

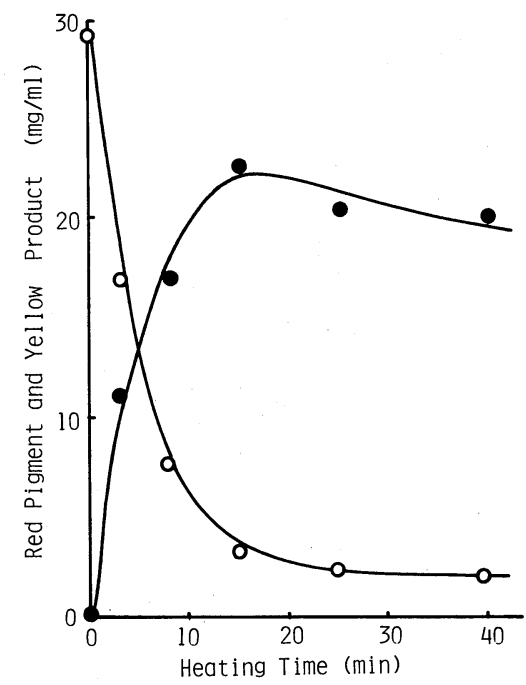

Fig. 5. Formation of Yellow Product by the Reaction of Red Pigment with SCA.

The mixture of red pigment and SCA $(0.1 \mathrm{M}$ each) in ethanol was refluxed in a boiling water bath. - yellow product; $-\mathrm{O}-$, red pigment.

produced was 5 times larger than that obtained by the DHA-Phe reaction system (see Fig. 2). In contrast with the latter system, substitution of the ethanol solvent by water did not eliminate the production of the yellow product, suggesting a close relationship between SCA and the yellow product.

Heating the red pigment alone, or with
DHA or AsA did not produce the yellow product, but the reaction of the red pigment with SCA did, as shown in Fig. 5. Its yield was comparable with that obtained by the DHASCA system. This led to the supposition that the yellow product in the DHA-Phe reaction mixture is the direct result of the reaction of the red pigment with SCA, both of which are produced in the initial stages of the DHA-Phe reaction system.

\section{Preparation of the yellow product}

Isolation of the yellow product from the reaction mixture of DHA and Phe could not be carried out in a straightforward manner because the mixture contained the precursor to the radical $\mathrm{R}-\mathrm{A}$, which behaved similarly to the yellow product in paper chromatography. Oxidation of this product with $p$-benzoquinone served to oxidize this to the red pigment, ${ }^{3)}$ which could be separated from the yellow product. The yellow product itself was immune to this oxidation, and details of the isolation and purification procedure are described in the experimental section.

\section{Structure of the yellow product}

Elemental analysis of the yellow product indicated the formula to be $\mathrm{C}_{18} \mathrm{H}_{22} \mathrm{O}_{15} \mathrm{~N}_{2}$. Considering that this product came from the result of Strecker degradation and should not have contained a residue coming from Phe, suggested the presence of three AsA or DHA residues in the molecule. Its UV absorption in water showed maxima at 250 and $370 \mathrm{~nm}$; the former seemed to indicate the involvement of at least one AsA residue.

The results of CMR spectroscopy are shown in Table II. From the nine higher field peaks, 62.75 to $88.23 \mathrm{ppm}$, the presence of three sets of carbons corresponding to C-4, C-5 and C-6 of the AsA or DHA residues is accepted as probable, in accordance with the elemental analysis. The values for the three pairs of signals A, B, C in the table were, respectively, close to those of C-6, C-5 and C-4 of the AsA ${ }^{7}$ or hydrated DHA molecule. ${ }^{8)}$ But those for the signals $73.60,75.95$ and $88.23 \mathrm{ppm}$ are in- 
TABLE II. PMR AND CMR DATA of THe Yellow Product

\begin{tabular}{|c|c|c|}
\hline Position* & $\delta_{\mathrm{H}(\mathrm{Hz})} * *$ & $\delta_{\mathrm{C}(\mathrm{OFR})} * *$ \\
\hline$A-6$ & $3.75(2 \mathrm{H}, \mathrm{d}, 6.6)\}$ & \multirow{2}{*}{$\mathrm{A}\left\{\begin{array}{l}62.75(\mathrm{t}) \\
63.22(\mathrm{t})\end{array}\right.$} \\
\hline$D_{1}-6$ & $3.72(2 \mathrm{H}, \mathrm{d}, 6.6)\}$ & \\
\hline \multirow[t]{2}{*}{$D_{2}-6$} & $4.12(1 \mathrm{H}, \mathrm{d}, 1.7)$ & \multirow[t]{2}{*}{$75.95(\mathrm{t})$} \\
\hline & $4.14(1 \mathrm{H}, \mathrm{d}, 3.8)$ & \\
\hline A-5 & $4.32(1 \mathrm{H}, \mathrm{td}, 6.6,1.2)\}$ & \multirow{2}{*}{$\mathrm{B}\left\{\begin{array}{l}69.73(\mathrm{~d}) \\
70.26(\mathrm{~d})\end{array}\right.$} \\
\hline$D_{1}-5$ & $4.09(1 \mathrm{H}, \mathrm{td}, 6.6,1.5)\}$ & \\
\hline$D_{2}-5$ & $4.70(1 \mathrm{H}, \mathrm{dd}, 3.8,1.7)$ & $88.23(\mathrm{~d})$ \\
\hline A-4 & $5.44(1 \mathrm{H}, \mathrm{d}, 1.2)\}$ & \multirow{2}{*}{$C\left\{\begin{array}{l}78.42(d) \\
81.42(d)\end{array}\right.$} \\
\hline$D_{1}-4$ & $4.63(1 \mathrm{H}, \mathrm{d}, 1.5)\}$ & \\
\hline $\mathrm{D}_{2}-4$ & $5.24(1 \mathrm{H}, \mathrm{s})$ & $73.60(d)$ \\
\hline$\left.D_{1}-3\right\}$ & & $\int 92.58(\mathrm{~s})$ \\
\hline $\left.\mathrm{D}_{2}-3\right\}$ & & $\{94.82(\mathrm{~s})$ \\
\hline $\mathrm{A}-2$ & & $117.87(\mathrm{~s})$ \\
\hline$A-3$ & & $140.82(\mathrm{~s})$ \\
\hline $\mathrm{D}_{1}-2$ & , & $159.19(\mathrm{~s})$ \\
\hline $\mathrm{D}_{2}-2$ & & $166.21(\mathrm{~s})$ \\
\hline A-1 & & $170.65(\mathrm{~s})$ \\
\hline$D_{1}-1$ & & $177.59(\mathrm{~s})$ \\
\hline $\mathrm{D}_{2}-1$ & & $187.02(\mathrm{~s})$ \\
\hline
\end{tabular}

* A, AsA moiety; $\mathrm{D}_{1}$, hydrated DHA moiety; $\mathrm{D}_{2}$, cyclic DHA moiety.

** ppm with DSS reference, $\mathrm{D}_{2} \mathrm{O}$.

dicative of their correspondence to C-4, C-6 and C- 5 of a DHA molecule with a dehydrated cyclic structure (between C-6 and C-3). ${ }^{8 \text { ) }}$

On the basis of the above supposition, the C-2 of the AsA residue(s) should correspond to the signal $117.87 \mathrm{ppm}$, among the signals 92.58, 94.82 and $117.87 \mathrm{ppm}$. Among the six signals 140.82 to $187.02 \mathrm{ppm}$, four should have corresponded to the three $\mathrm{C}-1 \mathrm{~s}$, and to the $\mathrm{C}-3$ of the AsA residue. The remaining two signals at 140.82 to $187.02 \mathrm{ppm}$, and also 92.58 and $94.82 \mathrm{ppm}$, are considered to correspond to the two pairs of C-2s and C-3s of the two DHA residues. $^{8)}$ Two signals among these were found in the region of 92.58 to $94.82 \mathrm{ppm}$, and the remainder in the region 140.82 to 166.21 ppm. Because the C-2 and C-3 of the DHA molecule give signals between 92 and $106 \mathrm{ppm}$, the two signals between 140.82 and 166.21 ppm should have been significantly shifted down field, if the above supposition was to have been justified. One possible explanation is that this shift was caused by the double $\mathrm{C}=\mathrm{N}$ bondings of $\mathrm{C}-2$ and $\mathrm{C}-3$ of the DHA molecule.

The results of PMR are also shown in Table II. On the basis of the results of decoupling experiments, the PMR signals were classified into three groups, i.e., (1) 3.75, 4.32 and 5.44 ppm, (2) 3.72, 4.09 and $4.63 \mathrm{ppm}$, and (3) 4.12, $4.14,4.70$ and $5.24 \mathrm{ppm}$. These groups respectively correspond to the protons in the three DHA (or DHA and AsA) residues containing the C-6, C-5 and C-4 protons. The two signals (4.12 and $4.14 \mathrm{ppm}$ ) in group (3) are considered to correspond to the two C-6 protons of one DHA residue with a dehydrated cyclic structure, although the spin coupling between these two was not observed because of the closeness of these signals to each other. Instead, spin coupling was seen between each of the two and the signal for the C-5 proton $(4.70 \mathrm{ppm})$. When the signals of group (1) are compared with those of C-4, C-5 and C- 6 of AsA ${ }^{8}{ }^{8}$ the former two were shifted downfield by 0.46 and $0.22 \mathrm{ppm}$, respectively. This seems to suggest an AsA-like structure which slightly differs from AsA for C-2 and C-3.

The above results can be rationalized by a structure for the yellow product composed of one AsA, one DHA with a cyclic structure and one hydrated DHA, residue, and in which two of the four $\mathrm{C}-2$ and $\mathrm{C}-3$ protons of the two DHA residues are doubly bonded. The structure also has two nitrogen atoms. Two alternative structures are possible to meet the above requirements.

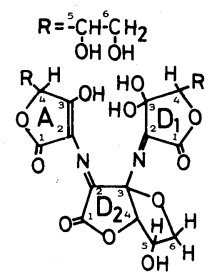

1

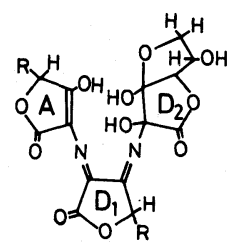

2
These structures differ in the presence or absence of a hydroxyl group on C-3 of the cyclic DHA residue in each. In other examples of DHA partial structures with a cyclic configuration, such as in DHA dimer ${ }^{8)}$ and in a tetrahydrocarboline derivative, ${ }^{9)} \mathrm{C}-3$ does not 


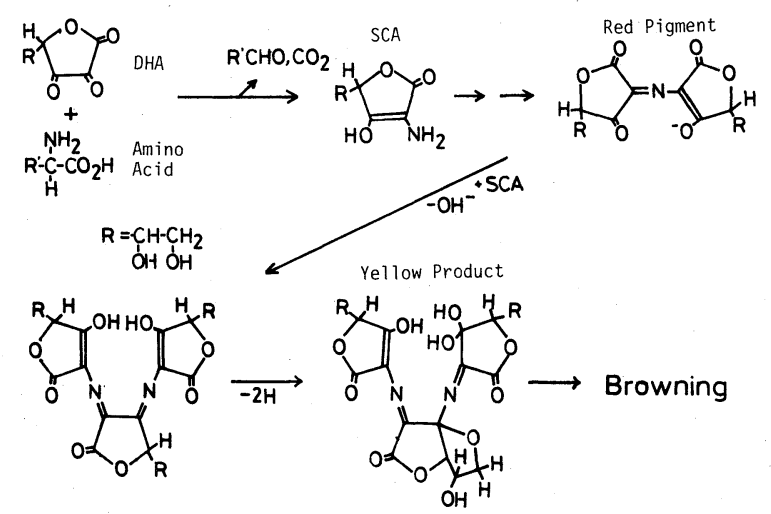

FIg. 6. A Possible Pathway for Formation of the Yellow Product.

have a hydroxyl group on it. This may perhaps indicate the tendency of a DHA structure to form the cyclic structure when the C-3 hydroxyl group is blocked. From this viewpoint the structure 1 may be a more probable one.

7. Path of formation of the yellow product and its significance in the browning reaction

The comments already made concerning the path of formation of the yellow product can be summarized as shown in Fig. 6. The condensation of two SCA molecules to C-2 and C3 of one DHA molecule can be regarded as analogous to the formation of DHA bishydrazone. ${ }^{10)}$

A noticeable fact is that the yellow product conceivably plays a very important role in the browning reaction of DHA with amino acids. This seems in accordance with the results of experiments in Table III, in which DHA, SCA, red pigment and the yellow product were each heated alone or in mixtures for $4 \mathrm{hr}$ in a $\mathrm{pH} 7$ aqueous solution, and the extent of browning was measured. Among these, the yellow substance alone or its mixture with DHA showed the highest degree of browning.

It is also to be noted that one of the free radical species produced by the DHA-amino acid reaction, $\mathrm{R}-\mathrm{B},{ }^{2)}$ was observed intensely by ESR in the reaction mixture just prior to the formation of the yellow product. This fact and the analysis of the ESR spectrum for R-B suggest that there exists a close relationship between R-B and the yellow product in the
Table III. Browning of an Aqueous Solution of the Yellow Product and Related Compounds by HeATING

\begin{tabular}{lc}
\hline \multicolumn{1}{c}{ Materials* } & Browning (O.D. at $420 \mathrm{~nm})$ \\
\hline DHA & 0.145 \\
DHA + Ala & 0.175 \\
SCA & 0.348 \\
SCA + DHA & 0.760 \\
Red Pigment & 1.40 \\
Red Pigment+SCA & 2.48 \\
Yellow Substance & 3.15 \\
Yellow Substance+Ala & 2.56 \\
Yellow Substance+DHA & 3.12
\end{tabular}

* Each solution (2.5 mM) in phosphate buffer $(0.1 \mathrm{M}$, $\mathrm{pH} 7$ ) was heated in a boiling water bath for $4 \mathrm{hr}$.

structure. A detailed analysis of the R-B formation is now being undertaken and will be presented in a following paper.

\section{EXPERIMENTAL}

Reagents. DHA was prepared in its dimeric form, by oxidation of AsA with $p$-benzoquinone. ${ }^{11)}$ SCA and the red pigment were prepared according to Kurata and others. ${ }^{1)}$ All other chemicals were of reagent grade.

TLC separation and analysis of products. For the separation of products, crystalline cellulose thin-layer plates (Merck plastic sheet) and an $n$-butanol-ethanol-wateracetic acid solvent system $(50: 20: 30: 1)$ were employed. The red pigment and the yellow product were visually detected at $R f=0.40$ and 0.36 , respectively. The SCA spot was visualized by spraying ninhydrin reagent and warming at $80^{\circ} \mathrm{C}$ for 1 to $2 \mathrm{~min}$. These spots were quantifieds using a chromatographic scanner (Shimadzu CS-900), by a zig-zag scan with the reference wavelength of $750 \mathrm{~nm}$. 
Since the spot for the yellow product was partially overlapped by that of the red pigment, a special procedure was necessary for the analysis of the former. The yellow product and red pigment have absorption maxima at 370 and $520 \mathrm{~nm}$, respectively, and the absorptivity of the latter at $370 \mathrm{~nm}$ is $1 / 1.6$ times of that at $520 \mathrm{~nm}$. In contrast, the absorption by the yellow product at $520 \mathrm{~nm}$ is negligibly small. Therefore the absorptivity of the spot at $370 \mathrm{~nm}$ minus that at $520 \mathrm{~nm}$ divided by 1.6 gives the absorptivity for the yellow product only, which can be used for the estimation of the yellow product using a calibration curve.

Preparation of the yellow product. A suspension of DHA dimer $(10 \mathrm{mmol}$ as the monomer) and Phe $(5 \mathrm{mmol})$ in $50 \mathrm{ml}$ of $95 \%$ ethanol was refluxed in a boiling water bath for $20 \mathrm{~min}$. After concentration, $50 \mathrm{ml}$ of water and a solution of $p$-benzoquinone $(1.1 \mathrm{~g})$ in ether were added, and the mixture was shaken for $5 \mathrm{~min}$. The aqueous layer was washed with fresh ether, freed from the ether in a vacuum and then freeze-dried. The dried residue was dissolved in ethanol and was chromatographed on a Sephadex LH-20 column $(3 \times 70 \mathrm{~cm}$, void volume $300 \mathrm{ml})$, with ethanol as the solvent. After removal of the reddish purple first eluate $(135 \mathrm{ml})$ the yellow fraction was collected $(100 \mathrm{ml})$. Its concentrate was further purified by preparative paper chromatography using $12(40 \times 40 \mathrm{~cm})$ sheets of Toyo Roshi no. 51A filter paper. The solvent was the same as that used in TLC experiments. The separated yellow band $(R f=0.25 \sim 0.3)$ was cut out and extracted with small quantity of water. Paper chromatography was repeated two times more to yield $50 \mathrm{mg}$ of yellow powder, which could not be crystallized. This yellow product was highly soluble in water, dimethyl sulfoxide and other alcoholic solvents but not in nonpolar solvents. It decomposed at $155 \sim 160^{\circ} \mathrm{C}$. Analysis: C, $41.33 ; \mathrm{H}, 4.06 ; \mathrm{N}$, $5.26 \%$. Calcd. for $\mathrm{C}_{18} \mathrm{H}_{22} \mathrm{O}_{15} \mathrm{~N}_{2}: \mathrm{C}, 42.69 ; \mathrm{H}, 4.35 ; \mathrm{N}$, $5.33 \%$. UV $\lambda_{\max }(\mathrm{nm})$ in water: $250(\varepsilon=15000), 370(\varepsilon=$
4300). IR ( $\mathrm{KBr}$ disk, $\mathrm{cm}^{-1}$ ): 1780 (lactone $v_{\mathrm{C}=\mathrm{o}}$ ), 1600 $\left(v_{\mathrm{C}=\mathrm{C}}\right)$.

Spectrometers. Spectroscopic instruments used were: a Hitachi 200-10 for UV and visible spectra, with a JNMMH-100 for PMR and JNM-FX-100 for CMR.

Acknowledgments. Helpful discussions and advice by Dr. Keiichi Tsuji of the Institute of Physical and Chemical Research are greatly appreciated. This work was supported in part by a Grant-in-Aid for Scientific Research from the Ministry of Education, Science and Culture of Japan.

\section{REFERENCES}

1) T. Kurata, M. Fujimaki and Y. Sakurai, J. Agric. Food Chem., 21, 676 (1973).

2) M. Yano, T. Hayashi and M. Namiki, J. Agric. Food Chem., 24, 815 (1976).

3) T. Hayashi and M. Namiki, Tetrahedron Lett., 4467 (1979).

4) T. Hayashi, F. Manou, M. Namiki and K. Tsuji, Agric. Biol. Chem., 45, 711 (1981).

5) K. Tsuji, T. Hayashi and M. Namiki, Electrochimica Acta, 25, 605 (1980).

6) M. Yano, T. Hayashi and M. Namiki, Agric. Biol. Chem., 42, 2239 (1978).

7) D. J. Goldsmith, J. Org. Chem., 44, 658 (1979).

8) J. Hvoslef and B. Pederson, Acta Chem. Scand., B33, 503 (1979).

9) M. Namiki, T. Hayashi and A. Shigeta, Agric. Biol. Chem., 46, 1207 (1982).

10) V. H. Hasselquist, Arkiv. f. Kemi, 4, 369 (1952).

11) W. Muller-Mulat, Hoppe-Seyler's Z. Physiol. Chem., 351, 52 (1970). 